\title{
A VIMOS spectroscopy study of photometric variables and straggler candidates in $\omega$ Centauri $^{\star}$
}

\author{
M. Rozyczka ${ }^{1}$, J. Kaluzny ${ }^{1}$, P. Pietrukowicz ${ }^{1,2}$, W. Pych $^{1}$, M. Catelan ${ }^{2}$, and C. Contreras ${ }^{2}$ \\ ${ }^{1}$ Nicolaus Copernicus Astronomical Center, ul. Bartycka 18, 00-716 Warszawa, Poland \\ e-mail: mnr@camk.edu.pl \\ 2 Pontificia Universidad Católica de Chile, Departamento de Astronomía y Astrofísica, Av. Vicuña MacKenna 4860, $782-0436$ Macul, \\ Santiago, Chile
}

Received 8 July 2011 / Accepted 25 October 2011

\begin{abstract}
We report a spectroscopic study of 19 photometric variables and 55 blue, yellow, and red straggler candidates in the field of $\omega$ Centauri. We confirm the cluster membership of 18 variables and 54 straggler candidates. Velocity variations are detected in 22 objects, and another 17 objects are suspected to be velocity-variable. The velocities of 11 objects vary consistently with their photometric periods, allowing their mass functions to be calculated. Among them, we find no indication of the presence of a massive degenerate component. On the basis of both photometric and spectroscopic data, we estimate that the fraction of binaries among blue stragglers may be as high as 69 per cent.
\end{abstract}

Key words. binaries: close - binaries: spectroscopic - blue stragglers - globular clusters: individual: NGC 5139 ( $\omega$ Centauri)

\section{Introduction}

Globular clusters harbor a massive population of close binary systems with degenerate components (hereafter referred to as degenerate binaries), discovered primarily due to their X-ray emission (e.g. Pooley 2010, and references therein). Unfortunately, detailed studies of these interesting objects are difficult because their optical counterparts are often faint and hard to identify in the crowded environment (e.g. Verbunt et al. 2008). Many field $\mathrm{X}$-ray binaries, however, exhibit long periods of quiescence. The nature of a quiescent binary is betrayed by a low-amplitude optical modulation induced primarily by the ellipsoidal effect of its nondegenerate component, while a spectroscopic observer would see it as as a single-line system with a large orbital velocity $\left(K>150 \mathrm{~km} \mathrm{~s}^{-1}\right)$. Perhaps the most characteristic examples of such objects are X-ray novae which almost certainly harbor black holes. They would be distinguished by a mass function $f_{\mathrm{m}}=(m \sin i)^{3} /\left(m_{\text {bin }}\right)^{2}>2 M_{\odot}$, where $m$ and $m_{\text {bin }}$ stand, respectively, for the mass of the degenerate component and the total mass of the binary (Remillard \& McClintock 2006).

The first spectroscopic search for quiescent degenerate binaries in globular clusters was conducted by Rozyczka et al. (2010) based on a sample of short-period, low-amplitude optical variables with nearly sinusoidal light curves and periods shorter than $\sim 1.3$ days. The sample included four objects from NGC 6397 and seven objects from $\omega$ Centauri (NGC 5139). The most interesting findings were turnoff binaries V17 and V36 in NGC 6397, whose invisible primary components had masses higher than $1 M_{\odot}$ (in the case of $\mathrm{V} 36$ even higher than $2 M_{\odot}$, albeit with a large uncertainty). In the present paper, we continue the search for degenerate binaries in $\omega$ Cen based on a sample of 20 optical

* Based on photometric data collected at Las Campanas Observatory, and spectroscopic data collected with the Very Large Telescope at European Southern Observatory (ESO programme 384.D-0736). variables with periods obtained by Kaluzny et al. (2004), which were classified by Bellini et al. (2009) as proper-motion members of that cluster.

Another class of cluster members that are certainly worthy of spectroscopic investigation consists of objects populating "nonstandard" areas of the color-magnitude diagram (CMD): blue stragglers, yellow stragglers, and sub-subgiants, the last of which are also called red stragglers (see e.g. Platais et al. 2011).

Blue stragglers were originally defined as stars located at the extension of the main sequence above the turnoff point. Since they are both brighter and hotter ("bluer") than turnoff stars, they appear to evolve at a slower rate than normal cluster members. Although they have been known for almost 50 years (Sandage 1953), the mechanism of their formation remains unclear. The various possibilities include mass transfer between the components of a binary, the merger of a binary, or the direct collisions of stars. It is also conceivable that all these mechanisms are at work, perhaps with various relative eficiencies in various clusters (Ferraro 2006; Ferraro et al. 2006; Dalessandro et al. 2008).

The results of Rozyczka et al. (2010) point to an extensive mass exchange rather than stellar collisions, as in all cases they report that the brighter component of the blue-straggler binary is the more massive primary star, which must have acquired significant amounts of hydrogen-rich material from its companion. Thus, if their sample is representative, then many blue stragglers must be Algol-like systems in which the original mass ratio has been reversed, causing the mass transfer to effectively stop. One of the most well-studied blue stragglers, star V228 in 47 Tuc is indeed a classical Algol (Kaluzny et al. 2007b); however, in other cases the similarity to Algols may be purely morphological. Another good example is the well-studied star V209 in $\omega$ Cen, which most probably underwent two mass transfer episodes. Its present primary seems to be "reborn" from a former white dwarf that accreted a new envelope through mass transfer from its companion, while the present secondary lost most of its 
envelope during the ascent along the subgiant branch, failed to ignite helium, and is now powered by a hydrogen-burning shell (Kaluzny et al. 2007a).

Yellow stragglers reside in the area between the subgiant branch and the horizontal branch. Most probably, they are evolutionary advanced blue stragglers (e.g. Xin et al. 2011), and distinguishing between these two groups of objects seems to be a matter of taste rather than physical necessity. Red stragglers are found to the right of the main sequence, below the subgiant branch. They are even less well-explored than the blue ones, and the conundrums they pose are even more mysterious (Platais et al. 2011).

Both the degenerate binaries and all kinds of stragglers are thought to be products of the evolution of binary systems in a dense stellar environment, and their peculiarities are most probably promoted or even induced by interactions between cluster members (Ferraro 2006; Platais et al. 2011). As such, they provide a link between classical stellar evolution and the dynamical evolution of the cluster, and represent a valuable observational template against which the dynamical models of stellar aggregates can be tested. The straggler-related goal of the present survey is to verify the membership of straggler candidates selected from the proper-motion catalogue of $\omega$ Cen by Bellini et al. (2009), and to establish how frequent binary systems are in the straggler population.

\section{Observations and data reduction}

We monitored selected targets in $\omega$ Cen with the help of VIMOS - a multi-purpose instrument mounted in the Nasmyth B focus of the ESO VLT-Unit 3 telescope. For the present survey, it operated as a multi-object spectrograph. To adapt it to the observations of blue stragglers, we selected the HR blue mode with wavelength range 4100-6300 A, resolution 2050-2550 (150-120 $\mathrm{km} \mathrm{s}^{-1}$ ) and dispersion $0.5 \AA /$ pixel.

A VIMOS spectroscopy run consists of pre-imaging and spectroscopic follow-up. The pre-imaging frames of $\omega$ Cen were obtained on Jan. 16, 2010 to serve as a basis for the preparation of masks with slits centered on objects chosen for the survey. The spectroscopic monitoring was performed over ten nights in February and March 2010. During each night, one $\sim 30$ min observation was made, consisting of acquisition imaging, two spectroscopic integrations of $580 \mathrm{~s}$ each, up to three flat-field exposures, and a helium-neon lamp exposure for wavelength calibration. The VIMOS field of view, which is composed of four $7 \times 8$ arcmin quadrants served by independent CCDs and separated by about 2 arcmin gaps, was centered on $(\alpha, \delta)_{2000}=\left(13^{\mathrm{h}} 26^{\mathrm{m}} 46.3^{\mathrm{s}},-47^{\circ} 27^{\prime} 51.8^{\prime \prime}\right)$. A log of the observations is presented in Table 1, in which date and airmass are given for the start of the exposures, and the seeing is averaged over each observation.

The target objects were selected from the variable star catalog of Kaluzny et al. (2004), henceforth identified by a number preceded with $\mathrm{V}$ or $\mathrm{NV}$, and the proper-motion catalog of Bellini et al. (2009), henceforth identified by a number preceded with B. We adopted the following selection criteria:

- a magnitude $V<19$ mag (which is the magnitude at which the signal-to-noise ratio expected for the latest spectral types in our sample amounts to $\sim 20$, assuming a $1^{\prime \prime}$ slit, $1^{\prime \prime}$ seeing, and a reasonably long exposure of $\sim 1000 \mathrm{~s}$ );

- the membership probability $m p$ in Bellini et al. (2009) should be at least $90 \%$;
Table 1. Log of observations.

\begin{tabular}{rccc}
\hline \hline ID & UT date & Airmass & Seeing $(\operatorname{arcsec})$ \\
\hline 1 & 20100211.32 & 1.11 & 2.0 \\
2 & 20100212.32 & 1.11 & 1.5 \\
3 & 20100213.31 & 1.12 & 1.0 \\
4 & 20100214.30 & 1.14 & 1.0 \\
5 & 20100221.26 & 1.19 & 1.0 \\
6 & 20100222.30 & 1.11 & 0.7 \\
7 & 20100223.25 & 1.19 & 0.7 \\
8 & 20100309.38 & 1.21 & 1.0 \\
9 & 20100310.30 & 1.09 & 1.3 \\
10 & 20100312.24 & 1.11 & 0.7 \\
\hline
\end{tabular}

- the image of the target should not blend with images of other objects on pre-imaging frames;

- the distribution of targets in pre-imaging frames should maximize the number of slits.

Photometrically detected pulsating stars, whose intrinsic variations if radial velocity could mask orbital effects, were excluded from the sample. The selection procedure was rather tedious, and in trying to maximize the number of photometric variables in the sample we were forced to relax the second criterion. After a few trials, we decided to focus on 81 objects consisting of 61 straggler candidates and 20 photometric variables, among which NV332, NV334, and NV361 had membership probabilities equal to $84 \%, 88 \%$, and $64 \%$, respectively. We deliberately included V209 - a system thoroughly investigated by Kaluzny et al. (2007a) - with the intention to use it as an indicator of the quality and reliability of velocity measurements. In the sample, there are a few blue objects that, strictly speaking, do not conform to the classical definition of blue stragglers, because they are located not on the main-sequence extension, but to the left of it. We included them to maximize the total number of slits.

Typical VIMOS spectra can be calibrated automatically by the ESO-VIMOS pipeline, which cannot, however, be entirely trusted because the spectra of some slits extend below $\lambda=$ $5000 \AA$, where calibration lamp lines are scarce $^{1}$, and some of them are very weak. Giuffrida et al. (2010) wrote an interactive procedure within the pipeline that provided more control of each calibration step; we decided to reduce the data manually, using standard IRAF ${ }^{2}$ procedures. We started from sci_raw frames and proceeded through object identification, slit extraction, flatfielding, science aperture extraction, lamp aperture extraction, wavelength calibration, and normalization of the spectra. Unfortunately, all flat-field images in quadrants 3 and 4 were contaminated by internal reflections. We were forced to substitute the affected image-sections with smooth fits, which, of course, degraded the quality of the corresponding sections of the spectra.

Seven slits produced no useful data. In five cases, either the target aperture could not be reliably extracted or the spectrum was too noisy, and the catalogued $(B-V)$ values of straggler candidates B283374 and B309015 turned out to be entirely inconsistent with their spectra, suggesting that those objects were either misidentified or very tightly blended. As a result, our sample decreased in size to 19 photometric variables and 55 straggler

1 ESO, 2011, online document at http://www.eso.org/ observing/dfo/quality/VIMOS/ServiceMode/ServiceMode. html

2 IRAF is distributed by the National Optical Astronomy Observatories, which are operated by the AURA, Inc., under cooperative agreement with the NSF. 


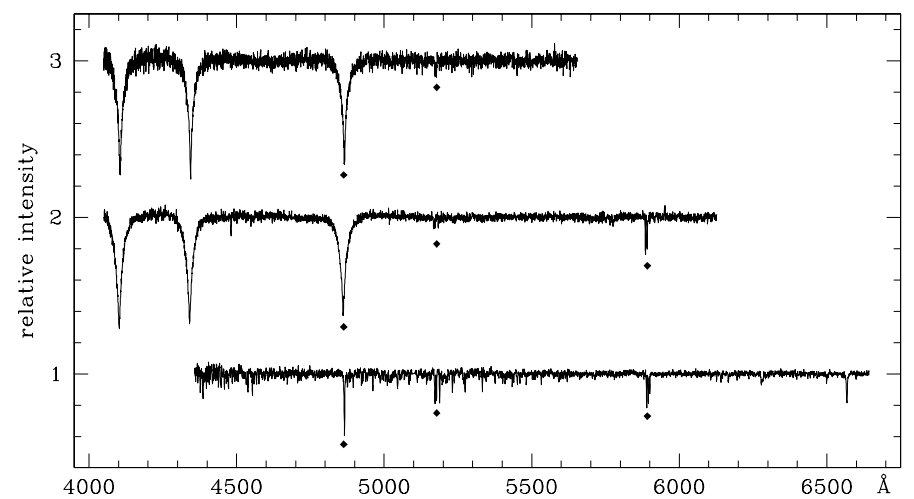

Fig. 1. Three examples of our spectra. The nominal spectral range of VIMOS in the setup used for the present survey begins at $4100 \AA$, but the recorded spectral range depends on the location of the slit on the mask and may extend shortward of that limit. From top to bottom, shown are: B289620 (shifted upward by 2), B319396 (aka V192; shifted upward by 1), and B155556 (aka V216). Diamonds mark lines used for velocity measurements: $\mathrm{H}_{\beta}$ at $4861 \AA$, magnesium triplet at 5167$5184 \AA$ A, and sodium doublet at 5890-5896 A.

candidates listed in Table 2. For each of them, we obtained ten reduced spectra. Three examples of the spectra are shown in Fig. 1. As detailed below, the information about our objects was mainly extracted from $\mathrm{H}_{\beta}$. The average $S / N$ at $\mathrm{H}_{\beta}$ was for all objects larger than 20 . Out of the total of 740 spectra, only a few had $S / N<20$.

A broad spectroscopic survey of $\omega$ Cen down to $V=$ 16.5 mag conducted by van Loon et al. (2007) at a resolution $R \sim 2000$ enabled us to verify the quality of the reduced spectra by a direct comparison. Among 15 of our variables and straggler candidates with $V<16.5$ we found four in common with their sample. In all four cases, the agreement was good - an example is shown in Fig. 2. We note that the nominal spectral range of VIMOS in the setup used for our observations starts at $4100 \AA$, while the spectra of van Loon et al. (2007) extend from $3840 \AA$ to $4940 \AA$, such that the range covered by both surveys is only $840 \AA$ in length.

\section{Analysis and results}

The actual spectral range recorded by VIMOS depends on the location of the slit on the mask. In our data, for all slits a wavelength region between 4700 and $5600 \AA$ was recorded, which in many spectra contained practically no lines except $\mathrm{H}_{\beta}$ and magnesium triplet at 5167.32, 5172.68 and 5183.60 $\AA$ (in the hottest objects even the latter was practically undetectable). The sodium doublet at 5889.95 and $5895.92 \AA$ were beyond the red wavelength limit of 11 spectra, and $\mathrm{H}_{\alpha}$ was visible in merely 13 spectra. Moreover, the accuracy of the wavelength calibration rapidly deteriorated with wavelength shortward of $4500 \AA$, where only two arc lamp lines (He 4471.48 $\AA$ and He 4026.19 $\AA$ ) could be used. Keeping this in mind and aiming to make our survey as uniform as possible, we decided to base the measurements on $\mathrm{H}_{\beta}$ fitting, using other methods for verification only.

The velocity was measured with the help of the IRAF task SPLOT by fitting Voigt profiles to $\mathrm{H}_{\beta}$. Wherever possible, it was

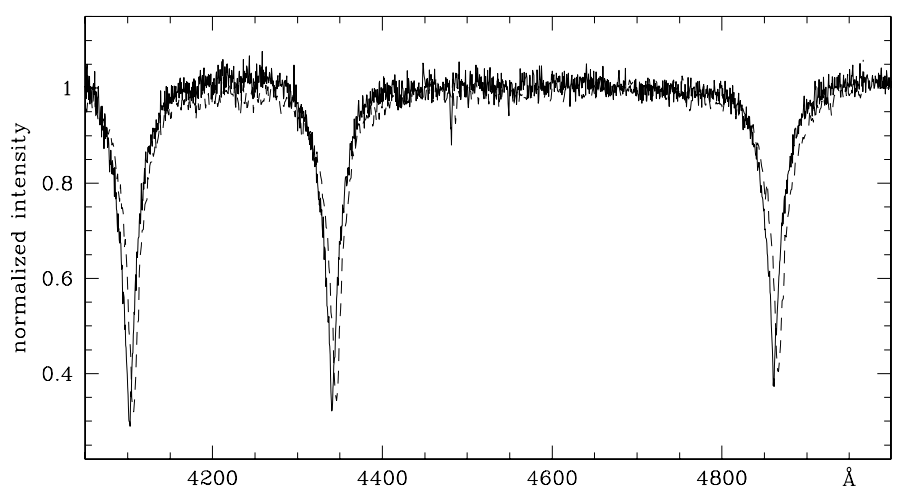

Fig. 2. A test of the quality of our spectra. Shown is the segment of the spectrum of B319396 (aka V192) common to the present survey (solid line) and that of van Loon et al. (2007) (broken line, shifted longwards by $5 \AA$ for clarity). Visible are $\mathrm{H}_{\delta}, \mathrm{H}_{\gamma}$, and $\mathrm{H}_{\beta}$.

also measured the same way from $\mathrm{Mg}$ and $\mathrm{Na}$ lines. For each line, the object velocity was calculated as a mean

$\bar{v}=\frac{1}{N} \sum_{i=1}^{N} v_{i}$,

where $N \leq 10$ is the number of fitted spectra, and the corresponding rms deviation

$\sigma=\sqrt{\frac{1}{N} \sum_{i=1}^{N}\left(\bar{v}-v_{i}\right)^{2}}$

was found. In the following, velocities and rms deviations are subscripted with the symbol of the element from which they are obtained, e.g. $\bar{v}_{\mathrm{H}}$ or $\sigma_{\mathrm{Na}}$. The results of sodium-based measurements are further differentiated by indices referring to the origin of the line, e.g. $\bar{v}_{\mathrm{Na}, \mathrm{i}}$ and $\bar{v}_{\mathrm{Na}, \mathrm{s}}$ are the velocities obtained, respectively, from the interstellar and stellar component of the line.

We also attempted to measure the velocities with the help of the IRAF task FXCOR. The measuring procedure consisted of the following steps:

- based on the calibration of Casagrande et al. (2010), the temperature $T$ of each object was estimated from the dereddened $(B-V)$ index assuming $E(B-V)=0.08($ McDonald et al. 2009);

- the temperature $T$ was rounded to the nearest multiple of $250 \mathrm{~K}$, and a corresponding template from the library compiled by Munari et al. (2005) was assigned to the object. Both $\log g$ and $[\mathrm{Fe} / \mathrm{H}]$ were the same for all templates and equal to 4.0 and -1.5 , respectively;

- each observed spectrum of the object was cross-correlated with the object's template.

The second step may seem oversimplified because of the large chemical composition spread in $\omega$ Cen (e.g. Johnson \& Pilachowski 2010) and the obvious spread of gravitational acceleration across our sample, in which $V$ varies by as much as 1.5 mag at constant $B-V$. In adition, the empirical formula of Casagrande et al. (2010) loses validity for $(B-V)<0.19$, i.e. for the eight hottest among our objects, and it may not be applicable to the 14 coolest objects lying far to the right of the main sequence. However, we checked on several objects that reasonable changes in $[\mathrm{Fe} / \mathrm{H}], \log g$ or even $T$ (i.e. by $\pm 0.5 \mathrm{dex}, \pm 0.5 \mathrm{dex}$, and $\pm 250 \mathrm{~K}$, respectively) did not modify the results of our velocity measurements in any significant way. 
Table 2. List of objects.

\begin{tabular}{|c|c|c|c|c|c|c|c|c|c|c|c|c|c|}
\hline & Bellini & $B-V$ & $V$ & VQ & $\bar{v}_{\mathrm{H} \beta}$ & $\sigma_{\mathrm{H} \beta}$ & $\bar{v}_{\mathrm{Na}}$ & $\sigma_{\mathrm{Na}}$ & Kaluzny & Period & $P_{\text {var }}$ & $V$ var & Remark \\
\hline 1 & 69263 & 0.36 & 18.33 & 1 & 227.5 & 12.7 & -5.7 & 9.2 & & & & & \\
\hline 2 & 69830 & 0.30 & 17.26 & 4 & 239.7 & 12.8 & 25.4 & 13.5 & & & & & \\
\hline 3 & 81157 & 0.22 & 17.23 & 1 & 210.6 & 34.9 & -55.7 & 12.8 & & & & $\mathrm{v}$ & \\
\hline 4 & 85380 & 0.25 & 16.94 & 1 & 230.4 & 12.4 & -50.8 & 17.1 & & & & & \\
\hline 5 & 87731 & 0.48 & 16.37 & 4 & 254.5 & 14.0 & 26.0 & 14.9 & & & & $\mathrm{~s}$ & \\
\hline 6 & 95154 & 0.32 & 16.75 & 4 & 256.9 & 15.9 & 2.7 & 6.4 & & & & $\mathrm{~s}$ & \\
\hline 7 & 95702 & 0.11 & 17.93 & 1 & 191.2 & 27.6 & -49.1 & 20.7 & & & & $\mathrm{v}$ & \\
\hline 8 & 99931 & 0.29 & 16.16 & 4 & 254.5 & 7.2 & 34.4 & 16.4 & & & & & \\
\hline 9 & 100977 & 0.39 & 16.73 & 1 & 239.7 & 12.0 & -43.4 & 13.7 & & & & $\mathrm{~s}$ & \\
\hline 10 & 107036 & 0.27 & 16.61 & 4 & 252.0 & 15.9 & 11.1 & 7.5 & & & & $\mathrm{~s}$ & \\
\hline 11 & 107122 & 0.32 & 17.87 & 1 & 202.9 & 16.6 & -47.0 & 15 & & & & & \\
\hline 12 & 111100 & 0.98 & 17.64 & 1 & 185.4 & 11.3 & -53.2 & 7.8 & & & & & lrs \\
\hline 13 & 113098 & 0.35 & 17.20 & 4 & 248.9 & 13.4 & 13.5 & 9.9 & & & & & \\
\hline 14 & 114490 & 0.28 & 17.78 & 1 & 195.6 & 14.7 & -48.8 & 10 & & & & & \\
\hline 15 & 118163 & 0.45 & 17.65 & 1 & 205.5 & 32.0 & -32.3 & 11.6 & NV337 & 0.269 & EW? & $\mathrm{v}$ & \\
\hline 16 & 121450 & 1.06 & 18.76 & 4 & 279.6 & 31.8 & 0.0 & 0 & & & & $\mathrm{v}$ & rs \\
\hline 17 & 121591 & 0.24 & 17.47 & 1 & 226.5 & 10.5 & -46.6 & 16.3 & & & & & \\
\hline 18 & 124330 & 0.49 & 16.07 & 1 & 81.2 & 66.1 & 0.0 & 0 & NV369 & 1.788 & ? & $\mathrm{v}$ & $\mathrm{nm}$ \\
\hline 19 & 130105 & 0.35 & 17.70 & 4 & 243.2 & 30.6 & 9.7 & 13.9 & NV401 & 0.354 & ? & $\mathrm{v}$ & \\
\hline 20 & 131682 & 0.47 & 16.68 & 1 & 197.9 & 10.2 & -52.1 & 10.7 & & & & $\mathrm{~s}$ & \\
\hline 21 & 133059 & 0.60 & 15.94 & 4 & 266.9 & 13.0 & 16.0 & 14.3 & & & & & \\
\hline 22 & 135321 & 0.99 & 18.83 & 1 & 232.6 & 10.4 & -20.6 & 27.3 & & & & & rs \\
\hline 23 & 137605 & 1.19 & 17.73 & 4 & 241.4 & 17.0 & 12.7 & 11.3 & NV332 & 0.247 & EW & & rs \\
\hline 24 & 140758 & 0.33 & 17.32 & 1 & 242.6 & 12.4 & -30.9 & 10.6 & & & & & \\
\hline 25 & 142842 & 0.33 & 17.26 & 4 & 241.4 & 30.7 & 2.4 & 11.2 & & & & & \\
\hline 26 & 143829 & 0.43 & 17.08 & 1 & 244.3 & 5.3 & -27.1 & 25.2 & & & & $\mathrm{~s}$ & \\
\hline 27 & 146095 & 0.20 & 17.58 & 4 & 256.9 & 64.1 & -7.6 & 11.9 & & & & $\mathrm{v}$ & \\
\hline 28 & 146967 & 1.10 & 17.98 & 1 & 262.7 & 35.6 & 13.1 & 45 & & & & $\mathrm{v}$ & lrs \\
\hline 29 & 148727 & 0.03 & 17.18 & 4 & 246.3 & 17.4 & -4.1 & 15.8 & NV380 & 7.832 & ? & & \\
\hline 30 & 149714 & 0.22 & 16.94 & 1 & 230.5 & 14.6 & -49.7 & 12 & & & & & \\
\hline 31 & 154483 & 0.10 & 16.74 & 1 & 228.9 & 13.7 & -49.4 & 9.9 & & & & & \\
\hline 32 & 155556 & 0.85 & 15.08 & 4 & 249.6 & 13.7 & 1.2 & 9.7 & V216 & 23.737 & LT & & $\mathrm{rg}$ \\
\hline 33 & 157688 & 0.25 & 17.95 & 1 & 225.6 & 13.7 & -40.4 & 10.2 & & & & & \\
\hline 34 & 159485 & 0.35 & 18.40 & 4 & 263.9 & 11.6 & 0.0 & 0 & & & & & \\
\hline 35 & 162119 & 0.99 & 17.63 & 1 & 219.7 & 12.6 & -52.8 & 9.1 & & & & & rs \\
\hline 36 & 166068 & 1.00 & 17.23 & 1 & 233.8 & 15.2 & -49.2 & 10.7 & & & & $\mathrm{~s}$ & rs \\
\hline 37 & 167555 & 1.03 & 16.38 & 4 & 272.3 & 15.5 & 15.3 & 13.9 & & & & & rs \\
\hline 38 & 171825 & 0.51 & 16.63 & 4 & 253.2 & 14.7 & 6.4 & 15.1 & & & & & \\
\hline 39 & 176840 & 0.25 & 16.92 & 1 & 229.2 & 15.8 & -43.7 & 8.4 & & & & & \\
\hline 40 & 177256 & 0.57 & 15.69 & 4 & 237.3 & 17.6 & -2.4 & 9 & V259 & 19.120 & $\mathrm{sp} ?$ & & \\
\hline 41 & 179912 & 0.32 & 16.44 & 1 & 229.8 & 23.0 & 0.0 & 0 & V239 & 1.189 & EA & $\mathrm{v}$ & \\
\hline 42 & 180526 & 0.97 & 17.78 & 4 & 250.4 & 16.2 & -35.0 & 11.6 & & & - & $\mathrm{s}$ & rs \\
\hline 43 & 234296 & 0.15 & 17.69 & 3 & 251.2 & 7.8 & 0.0 & 0 & & & & & \\
\hline 44 & 234998 & 0.39 & 18.20 & 2 & 237.4 & 24.5 & -2.1 & 15.5 & NV361 & 0.682 & EA & $\mathrm{v}$ & \\
\hline 45 & 241994 & 0.30 & 17.63 & 3 & 227.8 & 14.0 & 7.3 & 17.5 & & & & & \\
\hline 46 & 244408 & 0.41 & 17.01 & 2 & 248.0 & 28.9 & -12.3 & 14.8 & V208 & 0.306 & EW & $\mathrm{v}$ & \\
\hline 47 & 249955 & 0.49 & 16.51 & 2 & 222.0 & 13.4 & -13.9 & 15.7 & & & & $\mathrm{~s}$ & \\
\hline 48 & 250383 & 0.34 & 17.37 & 3 & 255.4 & 21.5 & 0.0 & 0 & & & & $\mathrm{v}$ & \\
\hline 49 & 253226 & 1.02 & 17.73 & 3 & 237.8 & 9.3 & 0.0 & 0 & & & & $\mathrm{~s}$ & rs \\
\hline 50 & 253281 & 1.00 & 18.55 & 2 & 225.2 & 22.1 & -24.0 & 25.4 & & & & $\mathrm{v}$ & rs \\
\hline 51 & 258274 & 0.49 & 16.88 & 2 & 206.9 & 27.0 & -21.3 & 17.3 & & & & $\mathrm{v}$ & \\
\hline 52 & 258539 & 0.18 & 16.84 & 3 & 241.0 & 10.8 & 19.3 & 8.4 & & & & $\mathrm{~s}$ & \\
\hline 53 & 262766 & 0.94 & 14.10 & 2 & 246.1 & 18.4 & -11.3 & 22 & NV390 & 15.710 & ? & $\mathrm{s}$ & $\mathrm{rg}$ \\
\hline 54 & 263560 & 0.37 & 17.30 & 3 & 207.3 & 18.4 & 9.8 & 14.3 & & & & $\mathrm{v}$ & \\
\hline 55 & 265591 & 0.29 & 17.29 & 2 & 219.1 & 19.7 & 0.0 & 0 & & & & & \\
\hline 56 & 269775 & 0.45 & 16.87 & 3 & 235.7 & 11.5 & 17.4 & 8 & & & & $\mathrm{~s}$ & \\
\hline 57 & 274338 & 0.18 & 16.98 & 2 & 237.3 & 6.8 & -13.1 & 17 & & & & $\mathrm{~s}$ & \\
\hline 58 & 277621 & 0.98 & 18.08 & 3 & 241.9 & 16.7 & -27.6 & 10.2 & & & & s & rs \\
\hline 59 & 279427 & 0.29 & 17.80 & 2 & 239.7 & 30.5 & 31.7 & 16.7 & & & & $\mathrm{v}$ & \\
\hline 60 & 281267 & 0.10 & 18.26 & 3 & 259.5 & 17.5 & 0.0 & 0 & & & & & \\
\hline 61 & 287148 & 0.28 & 17.33 & 3 & 230.0 & 15.0 & 16.0 & 11.2 & & & & $\mathrm{~s}$ & \\
\hline 62 & 289620 & 0.25 & 17.47 & 2 & 256.7 & 34.1 & 0.0 & 0 & & & & $\mathrm{v}$ & \\
\hline
\end{tabular}


Table 2. continued.

\begin{tabular}{cccccccccccccc}
\hline \hline & Bellini & $B-V$ & $V$ & $\mathrm{VQ}$ & $\bar{v}_{\mathrm{H} \beta}$ & $\sigma_{\mathrm{H} \beta}$ & $\bar{v}_{\mathrm{Na}}$ & $\sigma_{\mathrm{Na}}$ & Kaluzny & Period & $P$ var & $V$ var & Remark \\
\hline 63 & 293439 & 0.58 & 17.24 & 2 & 243.9 & 31.9 & -7.7 & 16.9 & V212 & 2.467 & EA & $\mathrm{V}$ & \\
64 & 297708 & 0.39 & 17.77 & 2 & 238.5 & 26.6 & -18.1 & 14.5 & V207 & 0.276 & EW & $\mathrm{v}$ & \\
65 & 297731 & 0.22 & 16.56 & 3 & 256.5 & 22.6 & 15.7 & 11.3 & V209 & 0.834 & EA & $\mathrm{v}$ & \\
66 & 302520 & 0.28 & 17.15 & 3 & 230.9 & 8.6 & 0.0 & 0 & V205 & 0.369 & EA & & nm \\
67 & 304775 & 0.52 & 15.77 & 3 & 44.4 & 15.9 & 35.0 & 8.9 & & & & & rs \\
68 & 306843 & 1.12 & 18.33 & 3 & 206.0 & 14.8 & 23.0 & 6.5 & & & & & \\
69 & 311900 & 0.64 & 15.12 & 2 & 228.5 & 17.0 & -11.9 & 11.1 & & & & $\mathrm{~s}$ & \\
70 & 312254 & 0.16 & 16.10 & 3 & 246.7 & 15.1 & 18.3 & 6.7 & $\mathrm{NV} 374$ & 3.311 & $?$ & & \\
71 & 317269 & 0.47 & 16.47 & 2 & 240.6 & 14.5 & -11.6 & 14 & & & & $\mathrm{~s}$ & \\
72 & 319396 & 0.22 & 16.01 & 2 & 241.9 & 17.0 & -12.7 & 11.1 & $\mathrm{~V} 192$ & 1.376 & $\mathrm{EA}$ & & \\
73 & 321474 & 0.62 & 17.86 & 3 & 256.6 & 74.6 & 7.5 & 16.4 & $\mathrm{NV} 363$ & 0.824 & $\mathrm{EA}$ & $\mathrm{v}$ & to \\
74 & 328605 & 0.84 & 16.19 & 3 & 233.8 & 19.8 & 14.9 & 6.9 & $\mathrm{NV} 379$ & 7.104 & $?$ & $\mathrm{v}$ & $\mathrm{rg}$ \\
\hline
\end{tabular}

Notes. Bellini - object's number in Bellini et al. (2009). $B-V$-color index, not corrected for reddening. $V-V$-band magnitude. VQ - number of VIMOS quadrant. $\bar{v}_{\mathrm{H} \beta}$ and $\sigma_{\mathrm{H} \beta}$ - mean value and rms deviation of velocity calculated from $\mathrm{H} \beta . \bar{v}_{\mathrm{Na}}$ and $\sigma_{\mathrm{Na}}-$ mean value and rms deviation of velocity calculated from the interstellar $\mathrm{NaI} \mathrm{D}_{2}$ line $(0$ if the spectrum did not reach to the NaI doublet). Kaluzny - object's number in Kaluzny et al. (2004). Period - photometric period in days. Pvar - type of photometric variability after Kaluzny et al. (2004): EA = Algol; EW = W UMa; $\mathrm{LT}=$ long term; $\mathrm{SP}=$ spotted. $V$ var - flag for velocity variability: $\mathrm{v}=$ variable; $\mathrm{s}=$ suspected. Remarks: $\mathrm{rs}=$ red straggler; lrs $=$ likely red straggler; $\mathrm{rg}=$ red giant; to = turnoff object; blank - object located to the left of main sequence and red giant branch (blue straggler, yellow straggler or hot subdwarf). The velocities are given in the heliocentric frame without corrections described in Sect. 3.3. The values of the corrections are 9.4, -3.4 , -6.6 and $21.0 \mathrm{~km} \mathrm{~s}^{-1}$ for quadrants $1-4$, respectively.

The spectral region selected for cross-correlation was located between $\mathrm{H} \beta$ and sodium doublet (including $\mathrm{H} \beta$ or more hydrogen lines resulted in extremely broad correlation peaks for hotter objects, whereas including sodium doublet introduced a signal from strong interstellar lines). It always contained the magnesium triplet, and its extent was chosen so as to maximize the correlation peak. Since in many hotter spectra the magnesium lines were not very much stronger than the noise, the resultant velocity measurement was considered to be satisfactory only if the maximum value of the correlation function $f_{\mathrm{m}}^{\mathrm{c}}$ exceeded 0.2 (for the synthetic template cross-correlated with itself $f_{\mathrm{m}}^{\mathrm{c}}$ was equal to 0.8 ).

\subsection{Discussion of errors}

The $\mathrm{H}_{\beta}$ line was fitted in all spectra of all objects, yielding 74 mean velocities $\bar{v}_{\mathrm{H}}$ and 74 corresponding rms deviations $\sigma_{\mathrm{H}}$. The latter originate from inaccurate fitting and/or calibration, and, in the case of binary systems, from the orbital motion. We checked that $\sigma_{\mathrm{H}}$ correlates with neither object magnitude, color, nor the location of the corresponding slit on the VIMOS mask (Fig. 3). This allows us to assume that fitting and calibration errors are purely random and have the same Gaussian distribution for all objects

$p(x)=\frac{1}{\sqrt{2 \pi s^{2}}} \exp \left(-\frac{x^{2}}{2 s^{2}}\right)$,

where $s$ is the (yet unknown) standard error in a single $\mathrm{H}_{\beta}$-measurement. Our observable $\sigma_{\mathrm{H}}$ is then obtained by randomly drawing ten values from the distribution given in Eq. (3), and calculating the root mean square

$\sigma_{\mathrm{H}}=\sqrt{\frac{1}{10} \sum_{i=1}^{10} x_{i}}$.

The expected distribution of the variable $y \equiv \sigma_{\mathrm{H}}^{2}$ is a chi-square with ten degrees of freedom

$p(y)=\frac{26.042}{s^{10}} \exp \left(-\frac{5 y}{s^{2}}\right) y^{4}$.
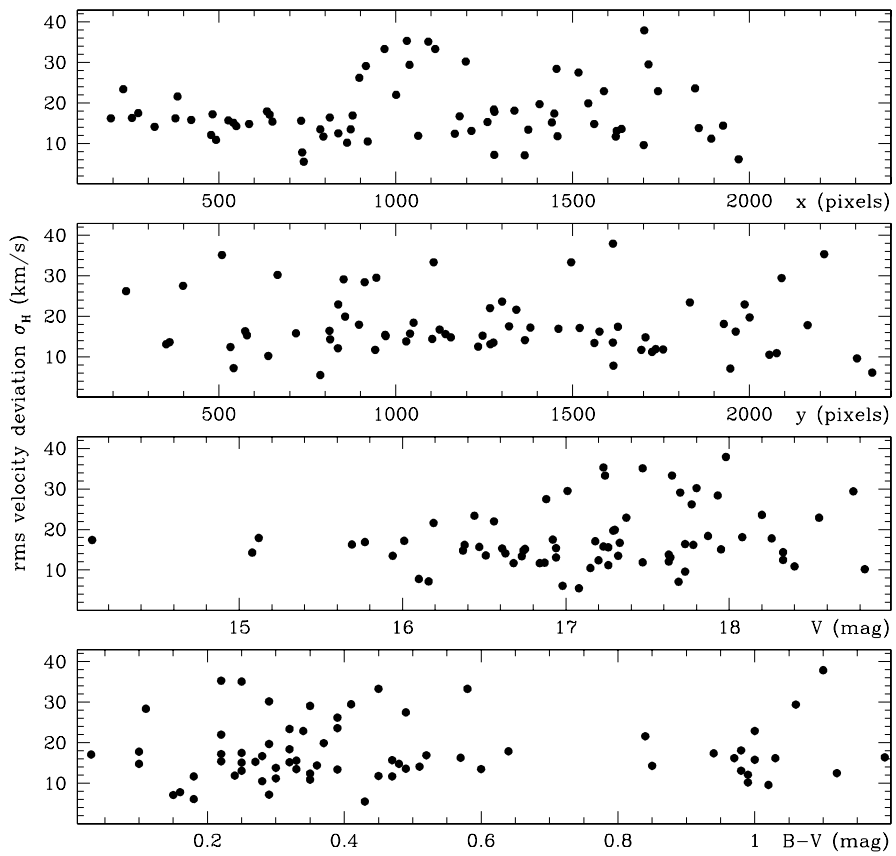

Fig. 3. Results of velocity measurements based on $\mathrm{H}_{\beta}$ fitting. The rms velocity deviation from the mean velocity of each object is plotted as a function of $(x, y)$ coordinates on the VIMOS mask, object's $V$ magnitude and object's color. Three objects with $\sigma_{\mathrm{H}}>40 \mathrm{~km} \mathrm{~s}^{-1}$ are not shown, which permits us to more clearly visualize the lower rmsrange.

In Fig. 4, the histogram of $y$ obtained from our measurements is compared to distributions of the form of Eq. (5) with $s=14,15$, and $16 \mathrm{~km} \mathrm{~s}^{-1}$. One can see that for $\sigma_{\mathrm{H}}^{2}<\sim 400 \mathrm{~km}^{2} \mathrm{~s}^{-2}$ the observed distribution is quite similar to the expected ones, and the high-velocity tail, clearly visible in the histogram but entirely absent from the expected distributions, is largely populated by short-period binaries. These two observations indicate that the assumptions about the random nature of the $\mathrm{H}_{\beta}$-measurement 


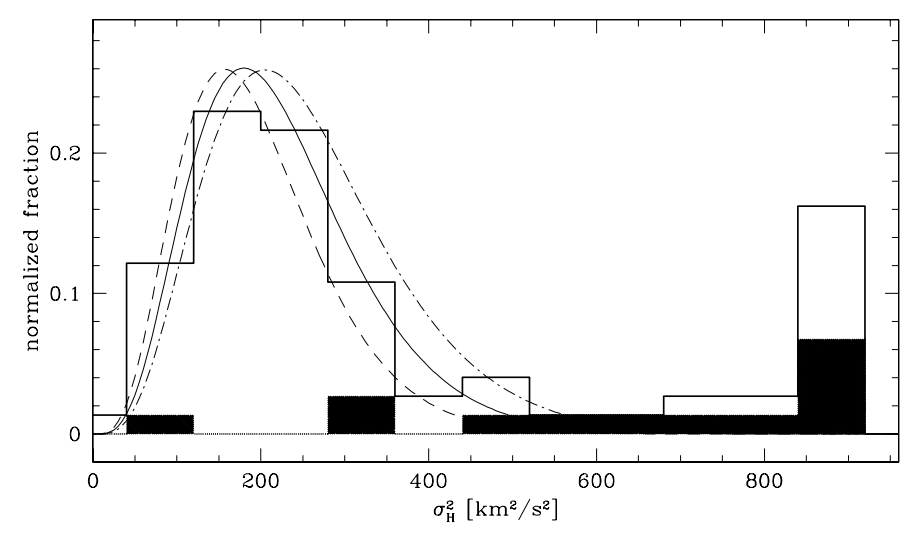

Fig. 4. Histogram of squared rms velocity deviations obtained from $\mathrm{H}_{\beta}$ fitting. Shaded are photometric variables with periods shorter than $3 \mathrm{~d}$. The expected distributions resulting from Eq. (5) for $s=14,15$, and $16 \mathrm{~km} \mathrm{~s}^{-1}$, where $s$ is the standard error in a single measurement, are shown with dashed, solid, and dot-dashed line, respectively. The rightmost bar contains all objects with $\sigma_{\mathrm{H}}>29 \mathrm{~km} \mathrm{~s}^{-1}$.

errors and the universality of their distribution are entirely reasonable. Among the three fits to the histogram the best one is that with $s=15 \mathrm{~km} \mathrm{~s}^{-1}$. Thus, we find that the standard error $\Delta v_{\mathrm{H}}$ in a single velocity measurement based on $\mathrm{H}_{\beta}$ fitting is equal to $15 \pm 0.5 \mathrm{~km} \mathrm{~s}^{-1}$. A similar accuracy of velocity measurements from VIMOS spectra (10-20 $\mathrm{km} \mathrm{s}^{-1}$ ) was reported by Giuffrida et al. (2010). A quantitatively similar, but intuitive rather than rigorous estimate of $\Delta v_{\mathrm{H}}$ follows from Fig. 5, in which the histogram of $\sigma_{\mathrm{H}}$ peaks at $\sim 15 \mathrm{~km} \mathrm{~s}^{-1}$.

The stellar Na doublet was usually blended with the interstellar one in such a way that the velocity could be reliably measured only from the interstellar $\mathrm{D}_{2}$ and the stellar $\mathrm{D}_{1}$ line (see also van Loon et al. 2007). The interstellar line was measured in all 63 objects whose spectra included the Na doublet, yielding the histogram of $\sigma_{\mathrm{Na}, \mathrm{i}}$ shown in Fig. 5. By analogy with the histogram of $\sigma_{\mathrm{H}}$, its central value of $\sim 13 \mathrm{~km} \mathrm{~s}^{-1}$ is a good estimate of the standard error in a single velocity measurement based on that line. It is slightly smaller than $\Delta v_{\mathrm{H}}$ because hydrogen lines are very broad in most objects, which results in larger fitting errors. In addition, the wavelength calibration is on average slightly less accurate at $\mathrm{H}_{\beta}$ than the $\mathrm{Na}$ doublet because there are fewer lamp lines in the bluer part of the spectrum.

We were able to derive a velocity measurement from magnesium lines for only 39 objects. In nearly all of them, the most clearly visible was MgI 5183.6 ̊. Unfortunately, for some objects even that line could not be well-fitted in all spectra, such that their $\bar{v}_{\mathrm{Mg}}$ and $\sigma_{\mathrm{Mg}}$ had to be calculated from as few as four spectra. As a result, the number of chi-square degrees of freedom was not uniquely determined for the $\sigma_{\mathrm{Mg}}$ observable. Owing to this, the histogram of $\sigma_{\mathrm{Mg}}$ in Fig. 5 is not directly comparable to the remaining two histograms. It was plotted for illustrative purpose only, and the rms deviations $\sigma_{\mathrm{Mg}}$ were not used in any further analysis. We only used the mean velocities $\bar{v}_{\mathrm{Mg}}$ to check whether they differ from their $\bar{v}_{\mathrm{H}}$ counterparts. No systematic differences were found, and the mean difference was equal to just $5.3 \mathrm{~km} \mathrm{~s}^{-1}$, which confirmed the reliability of mean-velocity measurements based on $\mathrm{H}_{\beta}$.

The FXCOR measurements fulfilled the reliability criterion $f_{\mathrm{c}}^{\mathrm{m}}>0.2$ in the case of 28 objects, while the stellar $\mathrm{Na} \mathrm{D}_{1}$ line was strong enough to permit a successful fitting in only $11 \mathrm{ob}-$ jects. As a result, there were only nine objects for which we collected four complete sets of independent velocity measurements

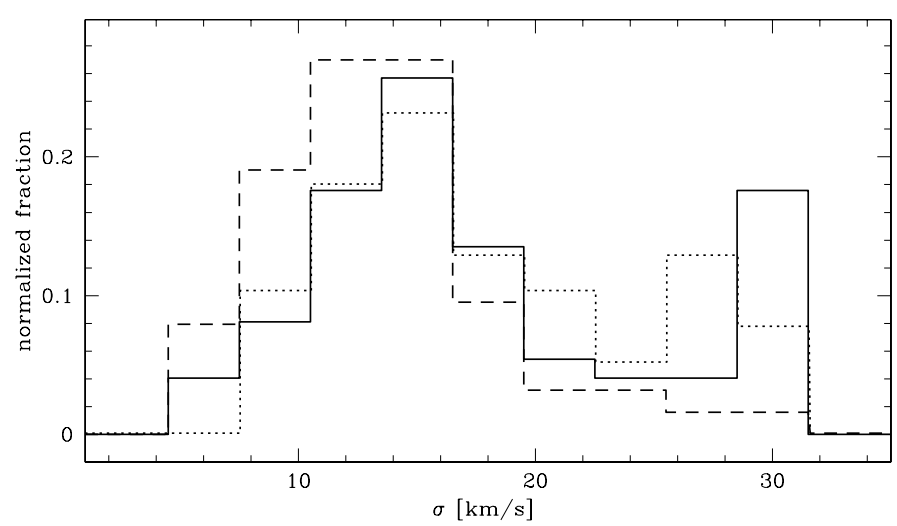

Fig. 5. Histograms of rms velocity deviations $\sigma_{\mathrm{H}}$ (solid), $\sigma_{\mathrm{Na}, \mathrm{i}}$ (dashed), and $\sigma_{\mathrm{Mg}}$ (dotted). The rightmost bar of each histogram contains all objects with $\sigma>\sim 29 \mathrm{~km} \mathrm{~s}^{-1}$. The $\sigma_{\mathrm{Na}, \mathrm{i}}$ measurements are based on the interstellar $\mathrm{D}_{2}$ line.

(from $\mathrm{H}_{\beta}, \mathrm{Mg}$ and stellar $\mathrm{Na}$ fitting, and from $\mathrm{FXCOR}$ ). For each spectrum of those objects, we calculated the average

$v_{4} \equiv 0.25\left(v_{\mathrm{H}}+v_{\mathrm{Mg}}+v_{\mathrm{Na}, \mathrm{s}}+v_{\mathrm{FXCOR}}\right)$

and the corresponding rms deviation $\sigma_{4}$. Figure 6 shows that there are no significant differences between $v_{4}$ and velocities obtained from $\mathrm{H}_{\beta}$ fitting, thus proving the reliability of the latter. All deviations $\sigma_{4}$ except one range between 2 and $20 \mathrm{~km} \mathrm{~s}^{-1}$.

We note that FXCOR returns formal velocity errors, but they are correct only to within a scaling factor that depends on the number of counts in the spectra and the Fourier filter parameters used (see e.g. For et al. 2010). For most of our objects, these errors turned out to be unrealistically small $\left(\sim 2-5 \mathrm{~km} \mathrm{~s}^{-1}\right)$.

\subsection{Velocity variables}

Figure 4 suggests that the lower limit to $\sigma_{\mathrm{H}}$ for the detection of radial-velocity (rv) variables is $\sim 20 \mathrm{~km} \mathrm{~s}^{-1}$. With this in mind, we werified whether the velocities of 11 photometric variables with $\sigma_{\mathrm{H}}>20 \mathrm{~km} \mathrm{~s}^{-1}$ are compatible with their lightcurves. We fitted the velocity data of each variable with a sinusoid using its photometric period $P_{\text {phot }}$ taken from Kaluzny et al. (2004). The only exception was V379, for which we used a period of $0.5 P_{\text {phot }}$, which is justified given the ambiguity of the lightcurve of Kaluzny et al. (2004).

The fitting was nearly successful - for the total of 110 points only three departed from the fits by more than $3 \Delta v_{\mathrm{H}}$ (two for NV337 and one for V208; see Fig. 7). In particular, we recovered velocity variations of our test-bed object - the V209 system, for which we had independent velocity data provided by Kaluzny et al. (2007a). From the fit in Fig. 7, we found that the velocity semi-amplitude of V209 is $32.5 \mathrm{~km} \mathrm{~s}^{-1}$, which closely matches the value of $30.7 \mathrm{~km} \mathrm{~s}^{-1}$ given by Kaluzny et al. (2007a) for the semi-amplitude of the primary component (note that the primary is four times brighter than the secondary, so that the contribution of the secondary to our spectra is rather unimportant).

On the basis of Figs. 4, 5, and 7, we conclude that objects with $\sigma_{\mathrm{H}}>20 \mathrm{~km} \mathrm{~s}^{-1}$ may be rather safely identified as genuine rv-variables. Among the straggler candidates without lightcurves, we found 11 these objects, which implies that there is a total of 22 rv-variables in the whole sample. Out of them six satisfied the $f_{\mathrm{m}}^{\mathrm{c}}>0.2$ criterion (four photometric variables and two straggler candidates), and in all those cases the results from FXCOR confirmed the rv-variability found from line fitting. 


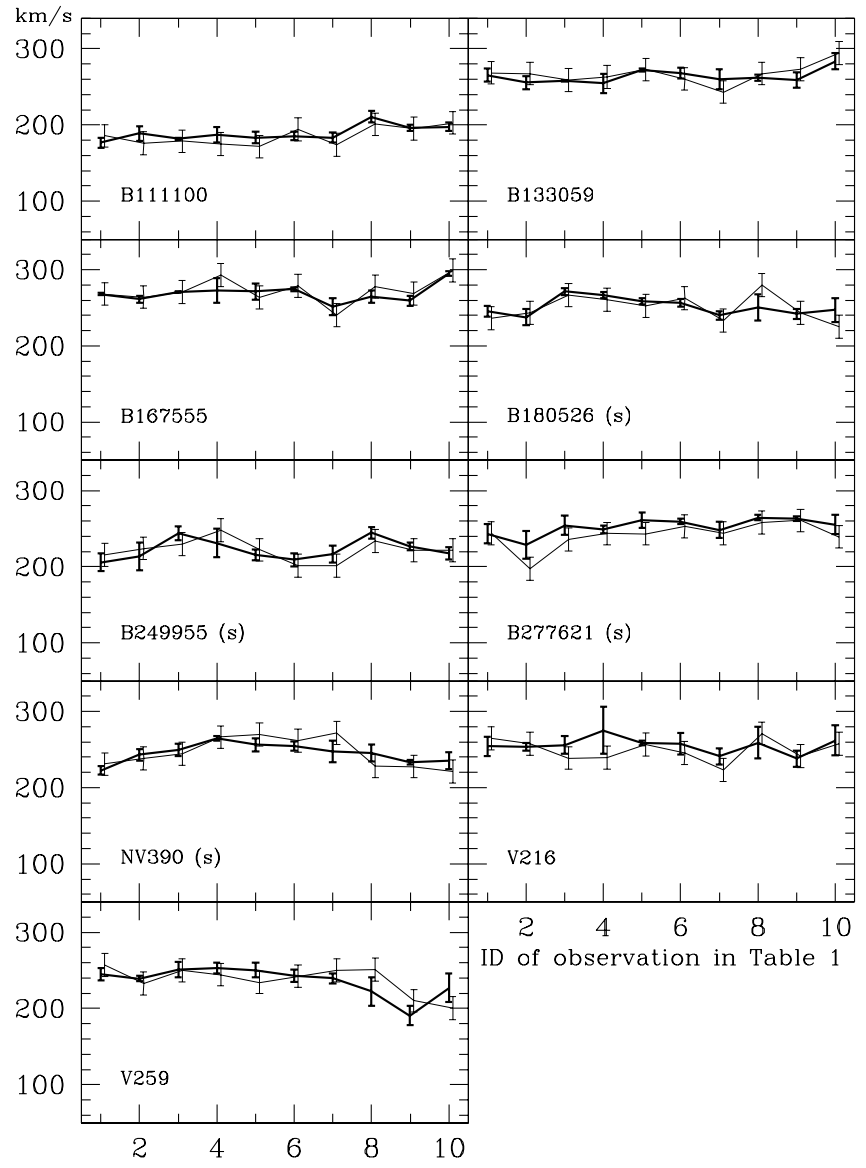

ID of observation in Table 1

Fig. 6. Velocities obtained from $\mathrm{H}_{\beta}$ fitting (thin lines) compared to averages $v_{4}$ defined by Eq. (6). Heavy errorbars are rms deviations from $v_{4}$. Thin errorbars (shifted by 0.1 to the right for clarity) mark the standard error in a single $\mathrm{H}_{\beta}$ measurement estimated in Sect. $3.1\left(15 \mathrm{~km} \mathrm{~s}^{-1}\right)$. Objects suspected of being velocity-variable (see Sect. 3.2) are indicated with an (s). All velocities are given in the heliocentric frame.

Additionally, we found 17 straggler candidates with $f_{\mathrm{m}}^{\mathrm{c}}>0.2$ showing consistent velocity variations whose full amplitude exceeded $30 \mathrm{~km} \mathrm{~s}^{-1}$, which we consider suspected rv-variables (see Table 2).

\subsection{Radial-velocity membership}

Using the IRAF task RVCORRECT, we transferred the velocities $\bar{v}_{\mathrm{H}}$ to the heliocentric frame. Their histogram is shown in Fig. 8a, where it is compared with the histogram of velocities measured for over 1500 stars in the field of $\omega$ Cen by van Loon et al. (2007). Before this comparison, a constant value of $-6.2 \mathrm{~km} \mathrm{~s}^{-1}$ was subtracted from the latter to account for the difference between the systemic velocity found by these authors $\left(238.3 \mathrm{~km} \mathrm{~s}^{-1}\right)$ and the value of $232.1 \mathrm{~km} \mathrm{~s}^{-1}$ given by Harris $(2010)^{3}$.

van Loon et al. (2007), who used an instrument where the field of view was served by two independent CCDs, found that on one of them the mean velocity of all stars $v^{\star}$ was $\sim 9 \mathrm{~km} \mathrm{~s}^{-1}$ larger than the other. They corrected for this effect by lowering

\footnotetext{
3 The comparison is allowable because our field of view and the central part of their field of view coincide, and the standard error in $\bar{v}_{\mathrm{H}}$, which is equal to $\Delta v_{\mathrm{H}} / \sqrt{10}$, is comparable to the velocity error reported in their survey $\left(\sim 5\right.$ versus $\left.\sim 8 \mathrm{~km} \mathrm{~s}^{-1}\right)$.
}

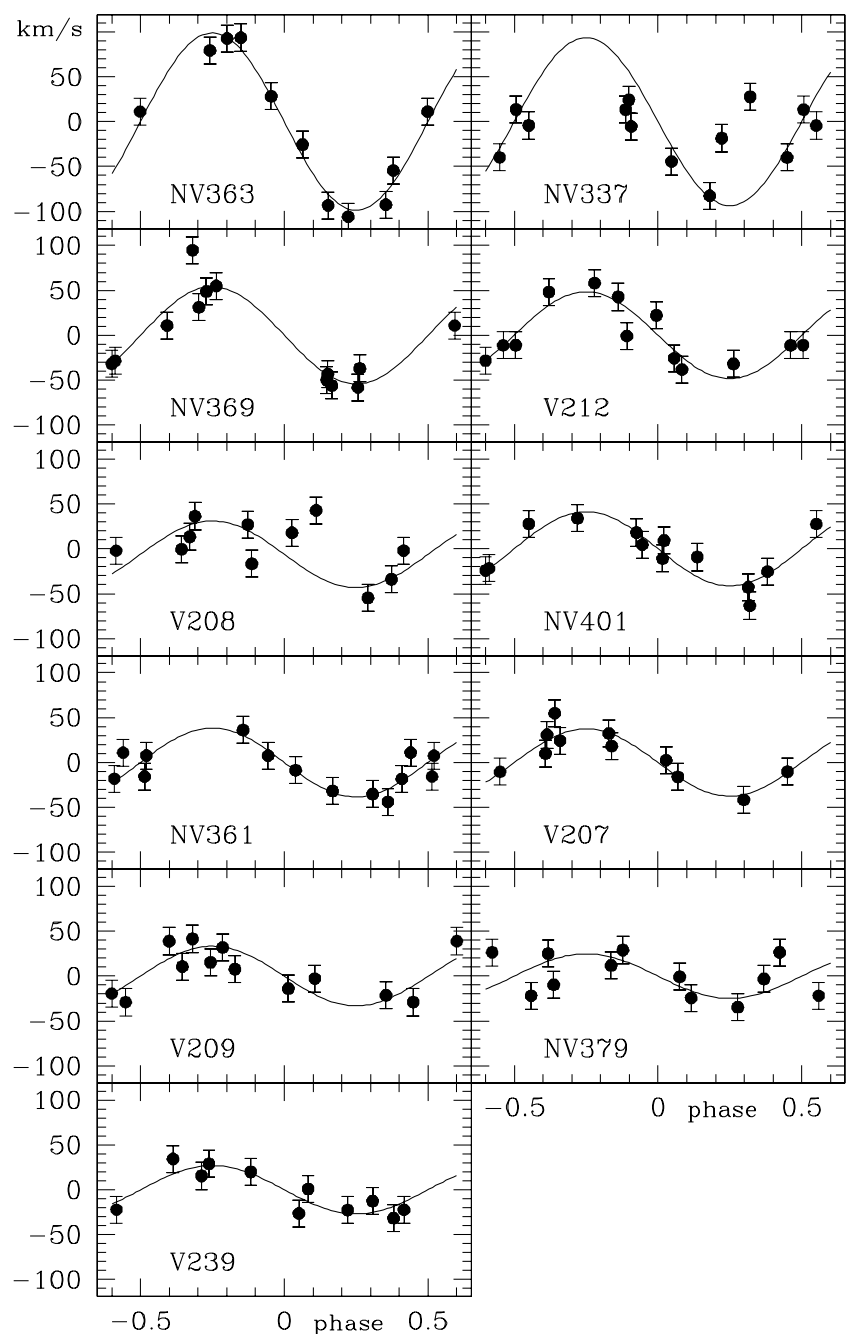

Fig. 7. Radial velocity curves of photometric variables. For each variable, the observational data (points) are phased with the corresponding photometric period $P_{\text {phot }}$ taken from Kaluzny et al. (2004). The only exception is NV379, where a period of $0.5 P_{\text {phot }}$ was used for phasing. Smooth curves are sinusoidal fits. Zero points on vertical axes correspond to systemic velocities. The standard error in the velocity measurement, estimated in Sect. 3.1, is $15 \mathrm{~km} \mathrm{~s}^{-1}$.

all radial velocities from the first CCD by $4 \mathrm{~km} \mathrm{~s}^{-1}$ and increasing all radial velocities from the second one by an equal amount. Expecting similar problems with our data from four CCDs, we performed the same test. Upon averaging $\bar{v}_{\mathrm{H}}$ from Table 2 over all stars in each quadrant, we obtained $v_{1}^{\star}=222.5, v_{2}^{\star}=235.5$, $v_{3}^{\star}=238.7$, and $v_{4}^{\star}=253.1 \mathrm{~km} \mathrm{~s}^{-1}$, respectively, for quadrants $1-4$. To account for these differences, we introduced corrections

$\Delta v_{i}^{\star} \equiv v_{\mathrm{sys}}-v_{i}^{\star}$,

where $v_{\text {sys }}$ is the systemic heliocentric velocity of $\omega$ Cen equal to $232.1 \mathrm{~km} \mathrm{~s}^{-1}$ (Harris 2010), and $i$ numbers the quadrants. We obtained $\Delta v_{1-4}^{\star}=9.4,-3.4,-6.6$, and $-21.0 \mathrm{~km} \mathrm{~s}^{-1}$.

The histogram of corrected velocities is shown in Fig. 8b, from which it is evident that our correction procedure makes sense. What remains to be explained are the large velocity deviations in quadrants 1 and 4 . Possible explanations include mask flexures (although one would expect their effect to be stochastic rather than systematic) and shifts in zero-point velocity caused by differences in the optical paths of light rays recorded by different CCDs. 


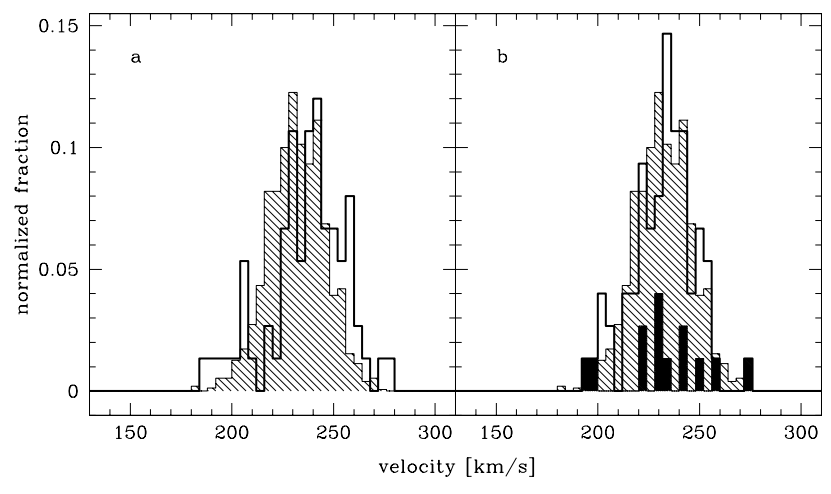

Fig. 8. Histograms of heliocentric $\bar{v}_{\mathrm{H}}$ velocities (heavy line) compared to the histogram of velocities obtained by van Loon et al. (2007) (shaded). a) $\bar{v}_{\mathrm{H}}$ uncorrected; b) $\bar{v}_{\mathrm{H}}$ corrected in such a way that the mean velocity of all objects in each VIMOS quadrant is equal to $232.1 \mathrm{~km} \mathrm{~s}^{-1}$ given by Harris (2010) as the systemic velocity of $\omega$ Cen. Black bars in Fig. b indicate red straggler candidates.

The minimum and maximum corrected heliocentric velocity of our targets was equal, respectively, to 195.1 and $272.4 \mathrm{~km} \mathrm{~s}^{-1}$ (minimum and maximum uncorrected velocity was, respectively, 185.4 and $279.6 \mathrm{~km} \mathrm{~s}^{-1}$ ). On the basis of the kinematic criterion of van Loon et al. (2007), who assign to $\omega$ Cen all stars with $180 \mathrm{~km} \mathrm{~s}^{-1} \leq v \leq 300 \mathrm{~km} \mathrm{~s}^{-1}$, we may say that almost all objects in our final sample do indeed belong to the cluster. The membership is firmly excluded only for NV369 and B304775, whose velocities, not shown in Fig. 8, fall well below $100 \mathrm{~km} \mathrm{~s}^{-1}$. We note that this conclusion is valid independently of whether we include corrections defined by Eq. (7).

\section{Discussion}

The most exciting goal of the present survey - the identification of massive low-luminosity members of $\omega$ Cen - has not been achieved. The lack of accurate ephemerides prevented us from repeating the detailed analysis performed on a smaller sample of $\omega$ Cen photometric variables by Rozyczka et al. (2010). However, no mass function higher than $f_{\mathrm{m}}=0.22$ was found, and in addition this particular value was obtained for NV369 - the only photometric variable from the sample whose membership was rejected by our analysis. The typical value of $f_{\mathrm{m}}$ for cluster members was below 0.05 , indicating rather low-mass companions to photometric variables with rv-variations.

For 72 of the 74 objects investigated here, our radial velocity measurements confirmed the proper-motion membership of $\omega$ Cen determined by Bellini et al. (2009). This conclusion does not depend on whether corrected or uncorrected velocities are used, as in both cases all rv-members fulfill the kinematic membership criterion of van Loon et al. (2007). The two proper-motion members of $\omega$ Cen, which turned out to be field objects, are B304775 $(m p=100 \%)$ and NV369 $(m p=94 \%)$, whose uncorrected heliocentric velocities were $44 \mathrm{~km} \mathrm{~s}^{-1}$ and $81 \mathrm{~km} \mathrm{~s}^{-1}$, respectively. On the other hand, the two objects with the lowest proper-motion membership probability, i.e. NV361 $(m p=64 \%)$ and NV332 $(m p=84 \%)$, turned out to be genuine members of $\omega$ Cen. As in only these four cases both proper-motion and radial-velocity data were needed to firmly establish the status of the object, our results indicate that the proper-motion catalog of Bellini et al. (2009) is highly ( $\sim 95 \%)$ reliable.

We found that 11 out of 19 photometric variables and 11 out of 55 straggler candidates were rv-variables. The possibility that some rv-variable straggler candidates might be single pulsating

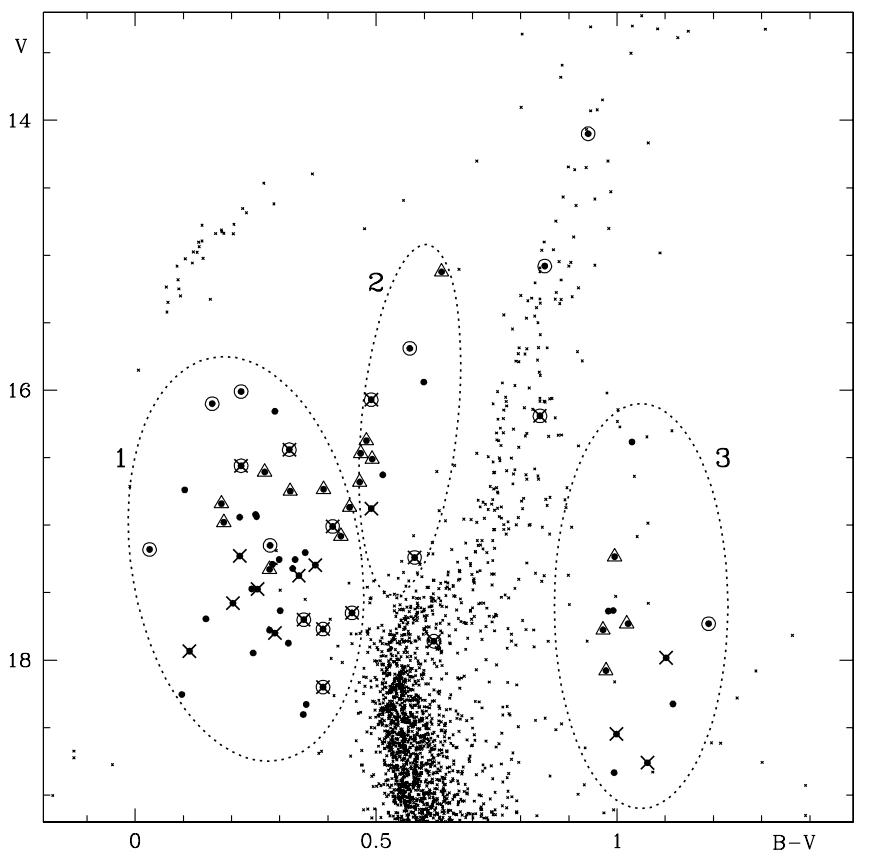

Fig. 9. Location of the investigated objects (large dots) in the CMD of $\omega$ Cen. Circles: photometric variables. Crosses: radial-velocity variables. Triangles: suspected radial-velocity variables. Ellipses 1, 2, and 3 indicate the approximate locations of the occupied regions, respectively, by blue, yellow, and red stragglers (see also Sect. 1). The background CMdiagram is from our unpublished photometry of a $2.6 \times 2.6$ arcmin field centered at $\alpha=13^{\mathrm{h}} 27^{\mathrm{m}} 42^{\mathrm{s}} .2, \delta=-47^{\circ} 23^{\prime} 34^{\prime \prime}$, obtained on the $2.5-\mathrm{m}$ duPont telescope at Las Campanas Observatory.

stars is excluded, as velocity variations of $\sigma_{\mathrm{H}}>20 \mathrm{~km} \mathrm{~s}^{-1}$ would cause detectable photometric effects in the Kaluzny et al. (2004) survey. Thus, our results indicate the binary nature of 22 objects. In addition, based on the cross-correlation of observed spectra with synthetic templates, we identified 17 straggler candidates suspected of being rv-variables. All these objects are shown in Fig. 9 in the CMD of $\omega$ Cen.

The high percentage of binary systems in the area occupied in Fig. 9 by blue and yellow stragglers allows us to firmly state that merging is not the only way of producing these objects. It is quite likely that all stars from our sample located in that region are binaries. The stars V192 and V205 at $(B-V, V)=(0.22$, $16.56),(0.28,17.15)$, for which we have not found any clear rvvariations, are eclipsing variables (Kaluzny et al. 2004). NV380, NV374 and V259 at $(0.03,17.18),(0.16,16.10)$ and $(0.57$, 15.69) have rather long periods $(7.83,3.31$ and $19.12 \mathrm{~d}$, respectively), and might have easily escaped recognition had their orbital inclinations not been close to $90^{\circ}$. If we number them among the suspected rv-variables, for the total of 51 cluster members located in the discussed region there will be 33 (i.e. $65 \%$ ) for which an indication of rv-variability was found. If we additionally include the binary blue stragglers discussed by Rozyczka et al. (2010), this ratio increases to $69 \%$.

Similar fractions of binary blue stragglers were found in two old open clusters: Mathieu \& Geller (2009) identified 16 binaries among 21 blue stragglers of NGC 188, and Latham (2007) found 8 binaries among 13 blue stragglers of M67. However, in both NGC 188 and M67 almost all binary blue stragglers have much longer periods than the objects discussed here - typically on the order of $\sim 1000$ days. Our spectroscopic survey lasted only two weeks, and it was quite insensitive to low velocities. As a result, we would not find any long-period variables even 
if they were there, and the lack of these objects in our sample may be a pure selection effect. However, the small percentage of short periods in NGC 188 and M67 compared to $\omega$ Cen is real, and must have a physical cause. Mathieu \& Geller (2009) pointed out that "the observation of binaries with circular orbits and periods of $\sim 1000 \mathrm{~d}$ in both NGC 188 and M67 indicates that these blue straggler binaries have not been disturbed dynamically since their formation". It is thus conceivable that the frequency of dynamical interactions in both open clusters is too low to cause efficient tightening of binary orbits, while in $\omega$ Cen it might be high enough to produce the observed shortperiod systems. In any case, that binaries are standard members of the blue and yellow straggler population is a strong argument in favor of the hypothesis that these objects were produced by a significant but rather quiet mass exchange in binary systems that rarely, if ever, led to merging. Our findings support the results of Ferraro et al. (2006), who, based on the flat radial distribution of blue stragglers in $\omega$ Cen, ruled out the collisional origin of these objects, and suggested that they are the progeny of primordial binaries. The same conclusion was reached by Dalessandro et al. (2008) in relation to NGC 2419.

Interesting objects have also been found to the right of the main sequence and below the subgiant branch of several clusters. They are the so-called red stragglers or sub-subgiants (e.g. Platais et al. 2011). As noted by Platais et al. (2011) in relation to NGC 6971, "these apparent binary stars occupy an area of the CMD ... which is not easy to populate with any combination of two normal cluster stars". At least some candidate red stragglers in $\omega$ Cen are X-ray sources, although the cause of their X-ray activity remains obscure (Haggard et al. 2010). The two binary red stragglers in M67 studied by Mathieu et al. (2003) may belong to the RS CVn class, but their parameters have not been uniquely determined. Mathieu et al. (2003) and Mathieu (2008) argue that their evolutionary history might have included mass transfer episodes, mergers, dynamical stellar exchanges, and/or close encounters leading to envelope stripping. In their conclusion, Mathieu et al. (2003) point to an intriguing possibility that these systems are the progenitors of blue stragglers.

According to the kinematic criterion of van Loon et al. (2007), who define $\omega$ Cen members as those with $180<v<$ $300 \mathrm{~km} \mathrm{~s}^{-1}$, all the 13 objects from our sample that reside to the right of the main sequence and red giant branch of the cluster, are members of the cluster (to be on the safe side, one may classify the three objects with extreme velocities as "likely members"; see Fig. 8 and Table 2). 12 red stragglers are subsubgiants, but the last one, B167555 at $(B-V, V)=(1.03,16.38)$, is less luminous by only $\sim 0.2 \mathrm{mag}$ than the photometrically and radial-velocity variable red giant NV379 at $(0.84,16.19)$, so that the alternative term "red straggler" fits it much better. Our red-straggler sample includes up to eight (i.e. up to $62 \%$ ) binaries: the eclipsing variable NV332 accompanied by the rvvariables B253281, B121450, and B146967 and four suspected rv-variables. It is thus conceivable that the remaining five objects are also binary systems, which would support the final conjecture of Mathieu et al. (2003). If that conjecture is right then red stragglers are relatively fresh products of complex interactions between cluster members, and as such they may turn out to be even more interesting than the blue stragglers themselves.

\section{Conclusions}

Our spectroscopic study of 74 proper-motion members of $\omega$ Cen has confirmed the membership of 72 objects. Among the 55 blue stragglers belonging to our sample, we found 22 binaries and

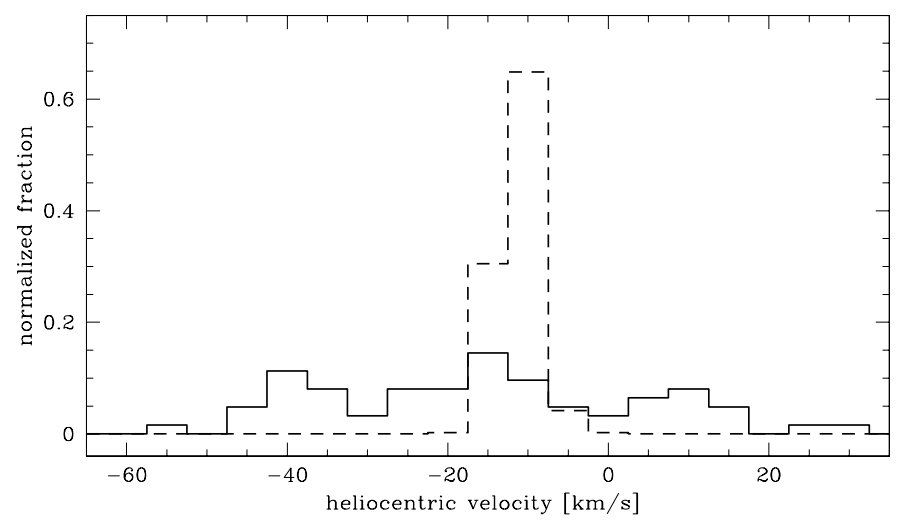

Fig. A.1. Histogram of heliocentric velocities obtained from $\mathrm{NaI} \mathrm{D}_{2}$ line. Solid: our data. Broken: van Loon et al. (2009).

13 suspected binaries, whereas among the 13 red stragglers we found four binaries and four suspected binaries. That binarity is normal in these intriguing populations leads one to expect that their evolutionary conundrums will be solved once good lightcurve and velocity-curve solutions are found. We hope that our results will prompt the relevant photometric and spectroscopic research.

Acknowledgements. Research of J.K., P.P., M.R., and W.P. is supported by the grant MISTRZ from the Foundation for the Polish Science and by the grants N N203 379936 and N N203 301335 from the Polish Ministry of Science and Higher Education. Support for MC and CC is provided by the Ministry for the Development, Econmy and Turism Programa Inicativa Científica Milenio through grant P07-021-F, awarded to The Milky Way Millenium Nucleus; by Proyecto Basal PFB-06/2007; by FONDAP Centro de Astrofísica 15010003; and by Proyecto FONDECYT Regular \#1110326. We are sincerely grateful to the anonymous referee whose detailed suggestions greatly improved the quality and the exposition of the paper.

\section{Appendix A: The baffling histogram of ISM velocity}

The globular cluster $\omega$ Cen is an ideal laboratory to study smallscale structure and dynamics of the interstellar medium (ISM) by using its stars as densely spaced beacons. Within the past two decades, there have been several attempts. Bates et al. (1992) and Wood \& Bates $(1993,1994)$ found several components of $\mathrm{NaI} \mathrm{D}_{2}$ at velocities between 0 and $-40 \mathrm{~km} \mathrm{~s}^{-1}$, "with the most conspicuous changes of the line profiles occurring in the most negative components, where variations were seen on an angular scale of $\sim 1$ arcmin". A strong patchiness of the absorbing medium was reported by Calamida et al. (2005), who registered "clumpy extinction variations by a factor of almost two across the core of the cluster". van Loon et al. (2007) detected a redshifted component of the CaII K line. Since it was visible only near the center of $\omega$ Cen, they suggested that it originated in a medium located within the gravitational influence of the cluster. High positive velocities were also measured in $\mathrm{HI}$ and $\mathrm{CO}$ emission lines (Origlia et al. 1997). A detailed mapping of interstellar clouds in front of $\omega$ Cen was more recently performed by van Loon et al. (2009). They found the ISM to be patchy on all scales from $0.7^{\circ}$ (the size of their field) down to about $30^{\prime \prime}$ (the closest distances between sightlines to sampled stars). The mean heliocentric velocity of the CaII $\mathrm{K}$ and $\mathrm{NaI} \mathrm{D}_{2}$ lines obtained from their survey is equal to -24 and $-11 \mathrm{~km} \mathrm{~s}^{-1}$, respectively. The histogram of heliocentric $\mathrm{NaI} \mathrm{D}_{2}$ velocities based on their online data peaks at $-11 \mathrm{~km} \mathrm{~s}^{-1}$, and is practically limited to the range between -6 and $-16 \mathrm{~km} \mathrm{~s}^{-1}$.

The above brief review indicates that the problem of the ISM in front of $\omega$ Cen is far from being solved, and this is what 


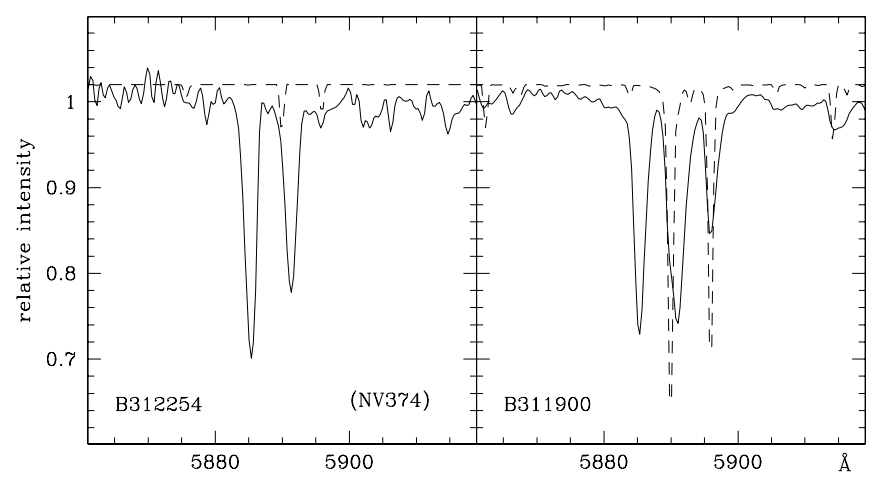

Fig. A.2. NaI doublet in Doppler-corrected spectra of B312254, aka NV374 $(B-V=0.16)$, and B311900 $(B-V=0.64)$. The observed spectra (solid lines) are compared to synthetic templates assigned as explained in Sect. 3.2 (broken lines). The templates are shifted upwards by 0.02 for clarity. Since the stellar spectra have been Doppler-corrected to the velocity obtained from $\mathrm{H}_{\beta}$, the ISM components appear shifted with respect to the rest wavelengths.

prompted us to extract the relevant information from our data. We measured the velocities of the $\mathrm{NaI} \mathrm{D}_{2}$ line, converted them into the heliocentric reference frame, and added the quadrantdependent corrections introcuced in Sect. 3.3. The resulting velocity histogram, shown in Fig. A.1, turns out to differ entirely from that of van Loon et al. (2009): the central maximum is much lower, broader, and shifted by $\sim 5 \mathrm{~km} \mathrm{~s}^{-1}$ toward more negative velocities. Moreover, an excess of objects with velocities between -35 and $-45 \mathrm{~km} \mathrm{~s}^{-1}$ and another one centered around $+10 \mathrm{~km} \mathrm{~s}^{-1}$ seem to be present.

Unfortunately, we do not have any objects common in with van Loon et al. (2009), such that a direct comparison of the spectra is impossible. Errors on our side could originate from incorrect velocity measurements, the misidentification of spectral lines, or a poor wavelength calibration. However, for each object $\bar{v}_{\mathrm{Na}}$ is an average over ten spectra. If true velocities were indeed strongly peaked at $-11 \mathrm{~km} \mathrm{~s}^{-1}$, then it would be difficult to imagine how these mean values could be wrong by as much as $\sim 30 \mathrm{~km} \mathrm{~s}^{-1}$ to produce the spurious peaks at -40 and $+10 \mathrm{~km} \mathrm{~s}^{-1}$. In addition, the identification of the NaI doublet is so easy that we can entirely exclude it as a source of errors. In hotter stars, there are no strong lines with which it could be mistaken, and in cooler stars the doublet turns into a unique triplet composed of interstellar $\mathrm{D}_{2}$ and stellar $\mathrm{D}_{1}$ with a blend of interstellar $\mathrm{D}_{1}$ and stellar $\mathrm{D}_{2}$ in between (Fig. A.2).

Shortwards of $\mathrm{H}_{\beta}$ our wavelentgh calibration is inaccurate in some objects ${ }^{4}$, but between $\mathrm{H}_{\beta}$ and $\mathrm{H}_{\alpha}$ it should be rather correct. We verifed this by selecting a few spectra that contain both of these lines, Doppler-correcting them for the velocity obtained from $\mathrm{H}_{\beta}$, and comparing with the corresponding synthetic templates assigned as explained in Sect. 3.2. In Doppler-corrected spectra, $\mathrm{H}_{\alpha}$ fell in the right place, and the stellar $\mathrm{D}_{1}$ line was also properly placed (Fig. A.2). We note that for a spurious shift of $\sim 30 \mathrm{~km} \mathrm{~s}^{-1}$ there is a relatively large calibration error of $\sim 0.3 \AA$, which should be easily visible in Doppler-corrected spectra. Smaller wavelength mismatches of course might have escaped out attention.

Another factor suspected of generating errors is instrumental effects (mask flexures, different zero-point velocities in different quadrants and/or slits). As a result, our velocity corrections introduced in Sect. 3.3 should vary not only between quadrants, but also between slits, and could not be calculated. One would have to accept that absolute velocities obtained from blue VIMOS spectra may be wrong by up to $\pm 30 \mathrm{~km} \mathrm{~s}^{-1}$. However, it was shown in Sect. 3.2 that the instrument is stable enough for reliable measurements of velocity variations with an accuracy of $\sim 15 \mathrm{~km} \mathrm{~s}^{-1}$.

\section{References}

Bates, B., Wood, K. D., Catney, M. G., \& Gilheany, S. 1992, MNRAS, 254, 221 Bellini, A., Piotto, G., Bedin, L. R., et al. 2009, A\&A, 493, 959

Calamida, A., Stetson, P. B., Bono, G., et al. 2005, ApJ, 634, L69

Casagrande, L., Ramírez, I., Meléndez, J., Bessell, M., \& Asplund, M. 2010, A\&A, 512, A54

Dalessandro, E., Lanzoni, M., Ferraro, F. R., et al. 2008, ApJ, 681, 311

Ferraro, F. R. 2006 [arXiv: astro-ph/0601217v1]

Ferraro, F. R., Sollima, A., Rood, R. T., et al. 2006, ApJ, 638, 433

For, B.-Q., Green, E. M., Fontaine, G., et al. 2010, ApJ, 708, 253

Giuffrida, G., Sbordone, L., Zaggia, S., et al. 2010, A\&A, 513, A62

Haggard, D., Cool, A. M., Arias, T., et al. 2010, AIPC, 1314, 157

Harris, W. E. 2010 [arXiv: 1012.3224]

Johnson, C. I., \& Pilachowski, C. A. 2010, ApJ, 722, 1373

Kaluzny, J., Olech, A., Thompson, I. B., et al. 2004, A\&A, 424, 1101

Kaluzny, J., Rucinski, S. M., Thompson, I. B., Pych, W., \& Krzeminski, W. 2007a, AJ, 133, 2457

Kaluzny, J., Thompson, I. B., Rucinski, S. M., et al. 2007b, AJ, 134, 541

Latham, D. W. 2007, Highlights Astron., 14, 444

Mathieu, R. D. 2008, IAUS, 246, 79

Mathieu, R. D., \& Geller, A. M. 2009, Nature, 462, 1032

Mathieu, R. D., van den Berg, M., Torres, G., et al. 2003, AJ, 125, 246

McDonald, I., van Loon, J. Th., Decin, L., et al. 2009, MNRAS, 394, 831

Munari, U., Sordo, R., Castelli, F., \& Zwitter, T. 2005, A\&A, 442, 1127

Origlia, L., Gredel, R., Ferraro, F. R., \& Fusi Pecci, F. 1997, MNRAS, 289, 948

Platais, I., Cudworth, K. M., Kozhurina-Platais, V., et al. 2011, ApJ, 733, L1

Pooley, D. 2010, PNAS, 107, 7164

Remillard, R. A., \& McClintock, J. E. 2006, ARA\&A, 44, 49

Rozyczka, M., Kaluzny, J., Pietrukowicz, P., et al. 2010, A\&A, 524, A78

Sandage, A. R. 1953, AJ, 58, 61

van Loon, J. Th., van Leeuven, F., Smalley, B., et al. 2007, MNRAS, 382, 1353

van Loon, J. Th., Smith, K. T., McDonald, I., et al. 2009, MNRAS, 399, 195

Verbunt, F., Pooley, D., \& Bassa, C. 2008, IAU Symp., 246, 301

Wood, K. D., \& Bates, B. 1993, ApJ, 417, 572

Wood, K. D., \& Bates, B. 1994, MNRAS, 267, 660

Xin, Y., Deng, L., de Grijs, R., \& Kroupa, P. 2011, MNRAS, 411, 761

\footnotetext{
4 ESO 2011, online document at http://www.eso.org/ observing/dfo/quality/VIMOS/ServiceMode/ServiceMode. html
}

A89, page 10 of 10 